\title{
Acceptance and Commitment Therapy to Support Medication Decision- making and Quality of Life in Women With Breast Cancer: Protocol for a Pilot Randomised Controlled Trial
}

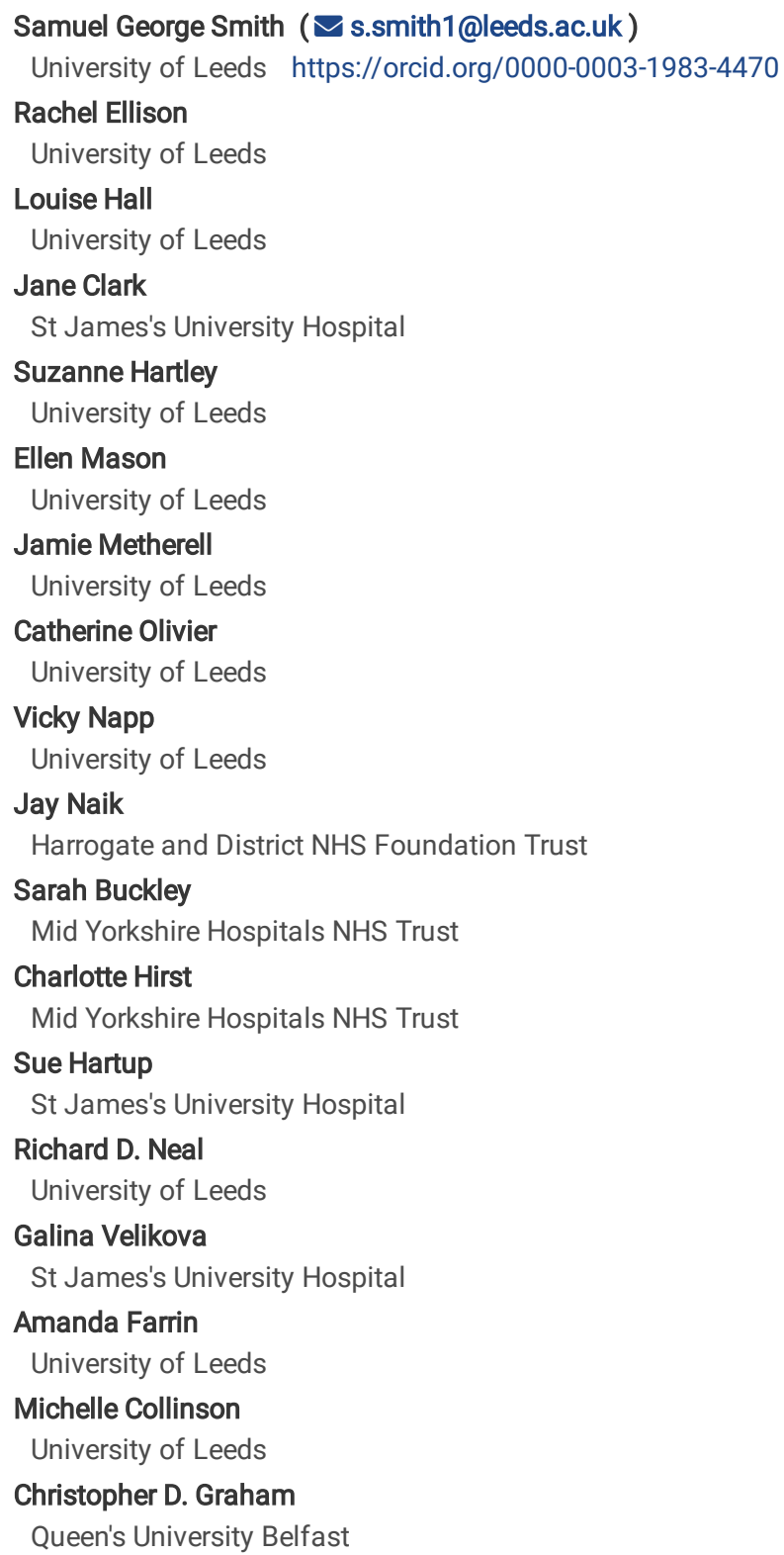

Study Protocol

Keywords: Medication adherence, breast cancer, Acceptance and Commitment Therapy, pilot, quality of life, remote delivery, group therapy, video conferencing

Posted Date: July 12th, 2021

DOI: https://doi.org/10.21203/rs.3.rs-679400/v1

License: (a) (1) This work is licensed under a Creative Commons Attribution 4.0 International License. Read Full License 
Version of Record: A version of this preprint was published at Pilot and Feasibility Studies on February 8th, 2022. See the published version at https://doi.org/10.1186/s40814-022-00985-6. 


\section{Abstract}

Background

Adherence to adjuvant endocrine therapy is affected by medication side-effects and associated distress. Previous interventions focused on educating women to enhance adherence have proved minimally effective. We co-designed an Acceptance and Commitment Therapy (ACT) intervention to enhance medication decision-making and quality of life by targeting a broader range of factors, including side-effect management and psychological flexibility. This study aims to establish key trial parameters, assess the acceptability of the intervention and the extent to which it can be delivered with fidelity, and to demonstrate "proof of principle" regarding its efficacy on primary and process outcomes.

Methods

The ACTION intervention includes an individual 1:1 ACT session followed by three group sessions involving 8-10 women and two practitioner psychologists. Participants are also provided with access to a website containing evidence-based methods for self-managing side-effects. The ACT sessions were adapted during the COVID-19 pandemic to be remotely delivered via video conferencing software. To evaluate the feasibility and acceptability of this intervention, a multi-site, exploratory, two-arm, individually randomised external pilot trial with a nested qualitative study will be undertaken. Eighty women with early stage breast cancer prescribed adjuvant endocrine therapy will be randomised (1:1) to receive treatment as usual or treatment as usual plus the ACTION intervention. The planned future primary outcome is medication adherence assessed by the ASK-12 measure. Progression to a phase III RCT will be based on criteria related to recruitment and follow-up rates, acceptability to patients, competency and fidelity of delivery, and proof of principle for change in medication adherence.

Discussion

This external pilot trial will be used to ascertain the feasibility of undertaking a future phase III RCT to definitively evaluate an ACT-based intervention to support medication taking behaviour and quality of life in women with early stage breast cancer on adjuvant endocrine therapy.

Trial registration

ISRCTN: 12027752. Reigstered 24 December 2020, https://doi.org/10.1186/ISRCTN12027752

\section{Background}

Breast cancer is the most commonly diagnosed cancer in women worldwide (1). In the UK there are approximately 55,000 cases occuring per annum, with 11,000 cancer-related deaths (2). Most breast cancers are oestrogen receptor positive (ER+) tumours. Treatment with adjuvant endocrine therapy (AET) such as Selective Oestrogen Receptor Modulators (SERMs e.g. tamoxifen) and Aromatase Inhibitors (Als e.g. letrozole, anastrozole, exemestane) for 5-10 years is standard care for women with ER + tumours (3). However, non-adherence is common with up to three-quarters of women prescribed adjuvant endocrine therapy $(\mathrm{HT})$ taking $<80 \%$ of prescribed therapy and similar proportions discontinuing prematurely (4). Low adherence and early cessation of AET are associated with an increased risk of breast cancer recurrence and all-cause mortality (5-12).

A broad range of behavioural and psychological variables such as mood, illness and treatment beliefs, and motivation are associated with adherence (13-15). Medication side-effects are also frequently reported to affect adherence to HTs (4). Common side-effects include hot flushes and night sweats, which affect up to $85 \%$ of women treated for breast cancer (16), and can compromise quality of life $(17,18)$. Several other frequently reported medication side-effects may also affect adherence to HTs, including joint pain, vulvovaginal symptoms and sleep disturbance (19). Most symptoms occur in the first year of using HT, suggesting this period could be the optimal opportunity for delivering supportive care for this patient group (20-22).

Adherence interventions evaluated so far have largely been ineffective (23-25). A meta-analysis of 8 interventions in this setting yielded a small to moderate heterogenous effect in favour of the interventions (24). The interventions largely focussed on educating patients regarding AET and solving problems with taking medication. These results, combined with evidence that a range of psychological factors contribute to adherence, led us to hypothesise that broadening the focus of an intervention to target emotions and self-management of side-effects could support adherence and quality of life in women who struggle with adhering to hormone therapy.

Acceptance and Commitment therapy (ACT) is a newer type of cognitive behaviour therapy that uses techniques such as mindfulness, goal setting, and perspective taking to engender a quality called psychological flexibility: "...the capacity to persist or to change behaviour in a way that includes conscious and open contact with thoughts and feelings, appreciates what the situation affords, and serves one's goals and values" (26). ACT may be a suitable approach for improving medication adherence following breast cancer for two main reasons $(13,27-30)$. First, psychological flexibility is a broad treatment target comprising a range of emotional and motivational factors that may contribute to non-adherence behaviours. Second, ACT is argued to be suited to helping people with health conditions. This is largely because ACT does not focus on changing understandable emotional responses to illness (e.g. frustration, anxiety etc.), and instead encourages finding ways to live well alongside these difficult experiences in order to fulfil life enriching goals and activity $(28,31)$.

We propose that ACT could be used to support functioning and medication adherence with women who have experienced a life-threatening event such as breast cancer, and are being asked to return to everyday life while managing treatment-related challenges. There is a growing evidence base supporting ACT as a means to improve functioning, quality of life, and mood in chronic disease and pain $(28,32)$. Although all have methodological limitations, 
several ACT trials with cancer populations have noted post-intervention improvements in outcomes, such as psychological distress and quality of life (28, 33, 33-36). There are no definitive trials of ACT for improving adherence to medication, with evidence limited to case series among people with diabetes and $\operatorname{HIV}(29,37,38)$.

We co-designed an intervention based on ACT principles through extensive engagement with patients and healthcare professionals (39). The aim was to co-design an intervention that was acceptable to patients and implementable within the United Kingdom (UK) National Health Service (NHS). In patient focus groups we explored women's experience of AET, perceptions regarding the acceptability of ACT, and preferences for intervention format. Healthcare professional interviews explored the acceptability and feasibility of an ACT intervention delivered within the NHS. In a co-design workshop, attended by patients and healthcare providers, we co-designed the configuration of the intervention. This process occurred prior to the coronavirus disease 19 (COVID19) pandemic. We altered the co-designed intervention so that it could be delivered via video-conferencing software instead of in person. The aim of the adaption was to ensure the ACTION intervention could be delivered in the context of potential future changes in how clinical psychology services will provide psychological interventions, and to ensure it could be delivered while social distancing guidelines were in place for a clinically vulnerable population.

The aim of the ACTION trial is to test the feasibility of undertaking a definitive phase III parallel groups Randomised Controlled Trial (RCT) of an ACTbased intervention for improving adherence to hormone therapy in women following curative treatment for breast cancer.

The objectives are to:

1. Establish eligibility, recruitment, retention and follow-up rates to inform the design of a phase III RCT;

2. Assess the acceptability of the intervention and protocol to participants and NHS therapists;

3. Assess the extent to which NHS therapists can remotely deliver an ACT intervention with fidelity, following remotely delivered training;

4. Demonstrate "proof-of-principle", via exploration of between-group change in outcomes (medication adherence, quality of life, mood) and process (psychological flexibility) variables.

\section{Methods}

\section{Design}

This is a multi-site, exploratory, two-arm, individually randomised pilot RCT with a nested qualitative study. Eighty women with early stage breast cancer will be randomised (1:1) to receive treatment as usual or treatment as usual plus the ACTION intervention. The study has been approved by the York and South Yorkshire Health Research Authority Research Ethics Committee (20/YH/0104), and is a registered clinical trial (ISRCTN12027752). The study is presented according to recommendations of the SPIRIT (40) and CONSORT extension for pilot and feasibility trials (41) (Appendix). The intervention being evaluated is described using the TIDieR checklist (42) (Appendix).

\section{Setting}

We will identify and recruit participants from oncology services at NHS hospitals in Yorkshire, UK. Sites with existing or planned implementation of a psychological intervention designed to improve adherence to HTs will be excluded. A single site will be responsible for delivering the intervention (Leeds Teaching Hospitals NHS Trust). Eligibility criteria for the delivery site include access to a Health and Care Professional Council (HCPC) registered practitioner psychologist (Clinical, Health or Counselling Psychologist) to deliver the intervention alongside at least one additional supporting member of staff (e.g. psychologist in training, assistant psychologist, counsellor, nurse therapist, or other HCPC registered staff). The delivery site must also have access to video conferencing software with the ability to create virtual breakout rooms, which can accommodate groups of 8-10 individuals.

\section{Participants}

Women aged over 18 and using tamoxifen, raloxifene, anastrozole, letrozole, or exemestane as part of their adjuvant endocrine therapy for early stage (1 to 3a) breast cancer are eligible. Full eligibility criteria are shown in Table 1. 
Table 1

Eligibility criteria for participation in the ACTION trial

1. Written (signed and dated) informed consent

2. Capacity to provide informed consent

3 . Women with early stage ( 1 to $3 a$ ) breast cancer according to the TNM / American Joint Committee on Cancer (AJCC) staging system

4. Aged $\geq 18$ years at time of screening for ACTION

5. Have sufficient proficiency in English to contribute to the therapy sessions and data collection required

6. Treated with curative intent

7. Completed their hospital-based treatment (e.g. surgery, radiotherapy and/or chemotherapy). Women are still eligible for the study before completing Herceptin.

8. Currently prescribed oral adjuvant Hormone Therapy (tamoxifen, raloxifene, anastrozole, letrozole, exemestane)

9. The participant is willing to be audio recorded during the therapy sessions

10. The participant is willing to complete the study questionnaires

11. The participant is willing and able to attend all intervention sessions and/or complete therapy workbook

12. The participant is willing and able to access all sessions remotely via video call
1. Stopped taking adjuvant hormone therapy if it is clinically contraindicated according to clinical recommendation

2. Currently or recently (last 6 months) involved in another psychotherapy (e.g. using CBT/ACT, mindfulness etc.) research study where medication adherence is a primary outcome

3. Those who are unable to access the sessions remotely via a video call

4. Currently attending, or on a waiting list for psychotherapy/psychooncology/psychology/counselling services, for any reason (related to medication or not)

5. Current diagnosis of an active major mental health disorder likely to interfere with participation (e.g. active psychosis, significant issues with addiction or selfharm)

6. Known element of risk (e.g. clinical team are aware that patient has made a recent attempt to end their life, or has recently disclosed plans to do so) as determined by three clinical screening questions below,

(i) Recently (in the last month), have you had any thoughts about ending your life?

(ii) Have you thought about how you might go about it?

(iii) Do you intend to carry out this plan?

* a patient is only excluded if they answer 'yes' to question 6 (iii)

\section{Study processes}

Participant identification can be via three routes:

1) A research nurse (or delegate) will screen patient records for eligible patients attending their final hospital appointment following completion of their hospital-based treatment;

2) An oncologist will screen patients discharged following treatment who self-refer to discuss problematic medication side effects and/or adherence problems;

3) A research nurse (or delegate) will retrospectively screen patient records for potentially eligible patients who have completed treatment within the last 6 months.

Sites will complete a screening form for all patients who have completed their hospital-based treatment for early stage breast cancer. The screening form will capture age, staging, ethnicity, and whether or not a patient is randomised. Screened patients who are not randomised will have the reason they are not eligible or declined participation recorded. Screening forms will not contain identifiable information.

For recruitment routes 1 and 2, a member of the patients care team will introduce a research nurse (or delegate) to the patient. For recruitment route 3 the research nurse or delegate will contact the patient by post. For all routes, a study invitation pack will be provided containing a participant information sheet, contact details, consent form, and a baseline questionnaire. Patients will have the opportunity to consent after considering the opportunity and asking questions, and may consent immediately once they are screened and confirmed as eligible. A research nurse will confirm eligibility among interested women, record consent, and ask the participant to complete the baseline questionnaire. Eligibility and consent discussions, and completion of baseline measures may take place in person at the hospital site or remotely. The site Principal Investigator (or delegate) is responsible for the informed consent of participants at their site.

Following informed consent, confirmation of eligibility and receipt of the baseline questionnaire, participants will be randomised by the research site using the University of Leeds Clinical Trials Research Unit (CTRU) randomisation system. Participants will be randomised on a 1:1 basis to receive either treatment as usual or treatment as usual plus the ACTION intervention and will be allocated a trial number. A computer-generated minimisation programme that incorporates a random element will be used to ensure treatment groups are well-balanced for the following stratification factors: recruiting site, recruitment route (recently completed treatment vs. experiencing medication problems vs. retrospective screening), participant age ( $\leq 50$ years vs. $>50$ years and $<70$ years vs. $\geq 70$ years).

Participants, therapists, research nurses/delegates at site, participant's GP and staff at the Clinical Trials Research Unit (CTRU) will be not be blinded to the randomised allocation. Following randomisation the research nurse will notify the participant of their allocation, and the therapists will be informed about participants allocated to the intervention arm. 
All participants will be requested to complete a follow-up questionnaire at 3 months post-randomisation. Participants recruited at least 3 months prior to the end of the recruitment period will also be invited to complete a questionnaire at 6 months post randomisation. Participants will have the option to receive questionnaires by post or online. Non-responders will be reminded using postal, telephone, online and text prompts.

A clinician may decide that a participant should be withdrawn from the intervention if there is reason to believe they have become unsuitable for the study. Participants can also withdraw from one or more of the following: intervention, data collected from them via questionnaires, or data about them from medical records.

\section{Treatment as usual}

All participants will receive treatment as usual which will be the standard care offered to women at this stage of their treatment for cancer. Women will be invited to an in person or remotely delivered end of treatment summary meeting with a breast cancer nurse involving a holistic needs assessment and provision of information relating to local services that can be accessed. Follow-up is patient-initiated, whereby the majority of patients are discharged from hospital follow-up, but are given contact details of a breast cancer nurse that they can speak with if they have any problems or concerns. The breast cancer nurse can make referrals to an oncologist, surgeon or other healthcare professional as needed. The content of treatment as usual received by participants will be collected for all participants in the trial.

\section{ACTION Intervention plus treatment as usual}

All participants in this group will receive treatment as usual in addition to the ACTION intervention. The ACTION intervention was co-designed with women affected by breast cancer and healthcare professionals. It is designed to enhance psychological flexibility as a means to improve AET adherence, and quality of life, while also being brief, and deliverable without significant changes to NHS services and staffing. The ACTION intervention is comprised of one individual ACT session ( 1 hour) followed by three group sessions (90 minutes each). Sessions will be delivered by practitioner psychologists remotely using locally approved videoconferencing software and will be digitally recorded, with the agreement of the participant. Within the first session, participants will also be directed to a bespoke ACTION website containing supplementary ACT exercises and additional information regarding HTs and advice on managing treatment side-effects. In addition a printable participant manual that includes all exercises to be undertaken in the four sessions will be sent to participants.

The ACTION intervention's format, aims and content are described in Table 2. The initial session is delivered by a practitioner psychologist, and they will be accompanied by a qualified member of staff for the group sessions. Access to home practice exercises will be via the ACTION website and in the participant manual. The manual will be sent to participants after the individual session by the therapists. The group sessions will include between $8-10$ participants, however they can run with a minimum of three confirmed attendees. A participant will be deemed to have received the intervention if they have attended the individual session and at least one of the three group sessions. 
Table 2

Format, aims and content of the ACTION intervention

\begin{tabular}{|c|c|c|}
\hline Session & Aim & Indicative Content \\
\hline $\begin{array}{l}\text { Individual } \\
\text { session } \\
\text { (60 minutes) }\end{array}$ & $\begin{array}{l}\text { - Assessment of psychological flexibility } \\
\text { - Relationship building } \\
\text { - Identify some clinically relevant behaviours } \\
\text { - Provide a simple formulation }\end{array}$ & $\begin{array}{l}\text { - Explanation of ACT and the ACTION intervention } \\
\text { - Conversations exploring } \\
\text { a) ongoing treatment and breast cancer experiences and how challenging } \\
\text { experiences are managed } \\
\text { b) where participants values might lie } \\
\text { - Communication of a psychological flexibility formulation } \\
\text { - Highlight the ACTION website including ideas for managing side-effects } \\
\text { Home Practice } \\
\text { - 'Getting to your core values' exercise } \\
\text { - 'What do you do with difficult feelings?' exercise }\end{array}$ \\
\hline $\begin{array}{l}\text { Group } \\
\text { session } 1 \\
\text { (90 minutes) }\end{array}$ & $\begin{array}{l}\text { - Enhance awareness of thoughts, feelings and } \\
\text { actions } \\
\text { - Introduce a values-based framework for } \\
\text { making decisions } \\
\text { - Introduce ways to relate to difficult thoughts } \\
\text { and feelings }\end{array}$ & $\begin{array}{l}\text { - Education: normalizing emotional responses to challenging events } \\
\text { - Discussion of 'What do you do with difficult feelings?' exercise } \\
\text { - Introducing defusion to support living with challenging thoughts: } \\
\text { - Experiential defusion practice e.g. } \\
\text { 'thoughts as hands' exercise } \\
\text { 'I am having the thought that...' exercise } \\
\text { Home Practice } \\
\text { - Defusion diary: noticing what thoughts tend to draw one in and practicing } \\
\text { defusing from them }\end{array}$ \\
\hline $\begin{array}{l}\text { Group } \\
\text { session } 2 \\
\text { (90 minutes) }\end{array}$ & $\begin{array}{l}\text { - Develop deeper awareness of values } \\
\text { - Consider hormone therapy decisions while } \\
\text { considering values } \\
\text { - Introduce willingness skills }\end{array}$ & $\begin{array}{l}\text { - Reflection on the 'Getting to your core values' exercise } \\
\text { - Values Compass } \\
\text { - Personal reflection on: 'what is the smallest possible step you could do to get in } \\
\text { touch with your values' } \\
\text { - Reflection on whether treatment adherence or side-effect management is a } \\
\text { values-consistent behaviour } \\
\text { - 'Passengers on the bus' exercise } \\
\text { Home Practice } \\
\text { Smallest possible step exercise } \\
\text { Defusion diary }\end{array}$ \\
\hline $\begin{array}{l}\text { Group } \\
\text { session } 3 \\
\text { (90 minutes) }\end{array}$ & $\begin{array}{l}\text { - Enhance awareness of getting caught up in } \\
\text { unhelpful stories } \\
\text { - Introduce ideas for stepping back from stories } \\
\text { - Reinforce new useful skills }\end{array}$ & $\begin{array}{l}\text { - 'Notice who is noticing' exercise } \\
\text { - 'Letting go of unhelpful labels' exercise } \\
\text { - Reflection on what has and has not been useful over the course of the } \\
\text { intervention. } \\
\text { - Reminder of the website including ideas for managing hormone therapy side } \\
\text { effects }\end{array}$ \\
\hline
\end{tabular}

The intervention was co-designed prior to the COVID-19 pandemic. We subsequently altered the method for delivering the intervention with support from patient representatives from face-to face delivery in a hospital setting to remote delivery using videoconferencing software. Participants are invited to attend from their own homes, while therapists are based at the hospital site or their home, in line with the standard practice for their clinical service.

Participants allocated to the ACTION intervention will be contacted by the psychology team delivering the treatment sessions. They will also be reminded about the ACTION website and encouraged to look at it prior to the session. The first session of the ACTION intervention will take place within 3 months of randomisation. The first group session will take place within six weeks of the first individual session.

\section{Participant Website}

The provision and content of a website was suggested by attendees of the co-design workshop (39), and designed by the research team with support from patient representatives. It contains sections on side-effect management, patient stories (via HealthTalk.org), ACT resources and exercises, and signposting 
for further information/support. The CTRU will email participants allocated to the ACTION intervention with log in details to the study specific website prior to the first session.

\section{Therapist training, competency and fidelity assessment}

As a novel intervention, we are particularly interested in designing training to enhance competency, and to assess the extent to which therapists deliver ACT with fidelity and deliver all ACTION study procedures.

\section{Therapist training}

All therapists will receive training in the ACTION intervention, which was designed and will be delivered by CG and JC. They are both registered clinical psychologists with experience in ACT and supporting women with breast cancer, respectively. Training will be delivered remotely over two full days via video conferencing software. The training will outline the psychological challenges of living with breast cancer and HTs, general ACT principles and the practice of ACTION intervention-specific therapy methods. To ensure consistency in intervention delivery, therapists will be given a comprehensive printable training manual and PowerPoint slides to support the group sessions. To assess whether therapists achieve sufficient knowledge of ACT principles, we will assess knowledge of ACT processes after training (43). They will be offered fortnightly ACT group supervision (60 minutes) with CG via video conferencing software, and can also access local clinical supervision (JC) as required.

A pool of therapists will be trained at the central delivery site to accommodate attrition. If a therapist is unavailable, efforts will be made to identify a second therapist from the pool of therapists. If no therapist is available from the pool, an additional therapist will be identified from within the clinical service. Prior to delivering an ACTION session, they will be asked to view a recording of the training sessions, and attend a 1-2 hour booster session with CG to ensure competency.

\section{Competency}

To support the establishment of competency CG will analyse the audio recording of the first individual session of all clinicians delivering the intervention, rate their performance against the ACT Fidelity Measure (ACT-FM) (44), and review the therapist checklist. The therapists will receive prompt feedback, and additional training will be offered where appropriate.

\section{ACT fidelity}

At the end of study, two independent assessors with expertise in ACT-based psychological interventions will listen to ten randomly selected therapy tapes and rate them for ACT fidelity using the ACT-FM. A minimum of one session will be reviewed from each therapist involved.

\section{Procedural fidelity}

We will also review therapist self-reports of fidelity to ACTION specific procedures via inspection of the Procedural Fidelity checklist.

\section{Measures}

The measures collected and their timing are illustrated in Tables 3 and 4. Primary and secondary endpoints are described in Table 5. 
Table 3

Summary of assessments and timing

\begin{tabular}{|c|c|c|c|c|c|c|}
\hline \multirow[t]{2}{*}{ Assessment } & \multirow[t]{2}{*}{ Source } & \multirow[t]{2}{*}{ Method of Completion } & \multicolumn{4}{|l|}{ TIMELINE } \\
\hline & & & Screening & Baseline & $\begin{array}{l}3 \\
\text { Months }\end{array}$ & $\begin{array}{l}6 \text { Months } \\
\text { (for patients recruited at } \\
\text { least } 3 \text { months prior to the } \\
\text { planned end of } \\
\text { recruitment) }\end{array}$ \\
\hline \multicolumn{7}{|l|}{ PARTICIPANT } \\
\hline Screening & $\begin{array}{l}\text { Screening } \\
\text { CRF }\end{array}$ & Research Nurse/Delegate & $x$ & & & \\
\hline Contact Details & CRF & Research Nurse/Delegate & & $x$ & & $\begin{array}{l}\text { CTRU to be notified of } \\
\text { address changes }\end{array}$ \\
\hline $\begin{array}{l}\text { Demographics including } \\
\text { comorbidities (Charlson Comorbidity } \\
\text { Index) }\end{array}$ & CRF & $\begin{array}{l}\text { Self- } \\
\text { completion/Research } \\
\text { Nurse/Delegate }\end{array}$ & & $\mathrm{x}$ & & \\
\hline $\begin{array}{l}\text { Eligibility } \\
\text { (including Inclusion and Exclusion } \\
\text { Criteria) }\end{array}$ & CRF & $\begin{array}{l}\text { Pl/Research Nurse/Pls } \\
\text { Delegate }\end{array}$ & & $\mathrm{x}$ & & \\
\hline Consent & $\begin{array}{l}\text { Consent } \\
\text { Form }\end{array}$ & $\begin{array}{l}\text { Self-completion / } \\
\text { RN/Delegate over the } \\
\text { telephone }\end{array}$ & & $x$ & & \\
\hline Randomisation & $\begin{array}{l}\text { CRF/CTRU } \\
\text { Online } \\
\text { system }\end{array}$ & Research Nurse/Delegate & & $\mathrm{X}$ & & \\
\hline ASK-12 & Questionnaire & Self-completion & & $x$ & $\mathrm{X}$ & $x$ \\
\hline McGill Quality of Life- Revised & Questionnaire & Self-completion & & $x$ & $\mathrm{X}$ & $x$ \\
\hline Work and Social Adjustment Scale & Questionnaire & Self-completion & & $x$ & $x$ & $x$ \\
\hline HFRDIS & Questionnaire & Self-completion & & $x$ & $x$ & $x$ \\
\hline MAF & Questionnaire & Self-completion & & $x$ & $x$ & $x$ \\
\hline PROMIS Pain Interference & Questionnaire & Self-completion & & $x$ & $x$ & $x$ \\
\hline DIVA (Part C) & Questionnaire & Self-completion & & $x$ & $x$ & $x$ \\
\hline GAD-7 & Questionnaire & Self-completion & & $x$ & $\mathrm{X}$ & $x$ \\
\hline PHQ-9 & Questionnaire & Self-completion & & $x$ & $x$ & $x$ \\
\hline FACT-ES & Questionnaire & Self-completion & & $x$ & $x$ & $x$ \\
\hline Valuing Questionnaire (VQ) & Questionnaire & Self-completion & & $x$ & $x$ & $x$ \\
\hline UK Cancer Costs & Questionnaire & Self-completion & & $x$ & $x$ & $x$ \\
\hline Acceptability & Questionnaire & Self-completion & & & & $x$ \\
\hline B \& N Questionnaire & Questionnaire & Self-completion & & & & $x$ \\
\hline Safety Reporting & CRF & $\begin{array}{l}\text { Research } \\
\text { Nurse/PI/Delegate/Self- } \\
\text { completion }\end{array}$ & & & $x$ & \\
\hline Usual Care Data & CRF & $\begin{array}{l}\text { Researcher/Delegate/ } \\
\text { Self-completion }\end{array}$ & & $x$ & $x$ & $x$ \\
\hline
\end{tabular}


Table 4

Summary of therapist, fidelity and competency assessments and timing

\begin{tabular}{|c|c|c|c|c|c|c|c|c|c|c|c|}
\hline \multirow[t]{2}{*}{ Assessment } & \multirow[t]{2}{*}{ Source } & \multirow{2}{*}{$\begin{array}{l}\text { Method of } \\
\text { Completion }\end{array}$} & \multicolumn{9}{|c|}{ TIMELINE } \\
\hline & & & $\begin{array}{l}\text { Pre- } \\
\text { therapist } \\
\text { training }\end{array}$ & $\begin{array}{l}\text { Post- } \\
\text { therapist } \\
\text { training }\end{array}$ & Baseline & $\begin{array}{l}\text { Individual } \\
\text { therapy } \\
\text { session }\end{array}$ & $\begin{array}{l}\text { 1st } \\
\text { Group } \\
\text { session }\end{array}$ & $\begin{array}{l}\text { 2nd } \\
\text { Group } \\
\text { session }\end{array}$ & $\begin{array}{l}\text { 3rd } \\
\text { Group } \\
\text { session }\end{array}$ & $\begin{array}{l}3 \\
\text { Month } \\
\text { follow } \\
\text { up }\end{array}$ & $\begin{array}{l}6 \\
\text { Month } \\
\text { follow } \\
\text { up }\end{array}$ \\
\hline \multicolumn{12}{|c|}{ THERAPIST } \\
\hline ACTKQ & Questionnaire & Therapist & & $x$ & & & & & & & \\
\hline Demographics & CRF & Therapist & & & $x$ & & & & & & \\
\hline ACT-FM & Questionnaire & $\begin{array}{l}\text { Independent } \\
\text { Expert } \\
\text { Reviewer }\end{array}$ & & & & & & & $x$ & & \\
\hline $\begin{array}{l}\text { Procedural } \\
\text { Fidelity } \\
\text { Checklist }\end{array}$ & CRF & Therapist & & & & $x$ & $x$ & $x$ & $x$ & & \\
\hline $\begin{array}{l}\text { Clinician } \\
\text { competency } \\
\text { using ACT- } \\
\text { FM* }^{\star}\end{array}$ & Questionnaire & $\begin{array}{l}\text { ACTION } \\
\text { Trainer }\end{array}$ & & & & $x$ & & & & & \\
\hline
\end{tabular}

Table 5

Primary and secondary endpoints recorded within the ACTION trial

\section{Primary Endpoints}

Number of patients screened for eligibility

Number and proportion of patients eligible out of those screened and reasons for ineligibility

Number and proportion of patients consenting to randomisation out of those eligible and reasons for non-randomisation

Number of participants randomised per site per month

Questionnaire completion rates at each time-point

Number of items of missing data per questionnaire at each time-point

Number and proportion of randomised participants lost-to-follow-up

Number, proportion, type and timing of participant withdrawals out of those randomised and reasons

for withdrawal

\section{Secondary endpoints}

Intervention adherence for patients in the intervention arm (number of sessions attended, number of participants in group sessions, whether homework was completed, reasons for nonattendance / non-compliance)

Website use (tracking data to include number of visits, pages visited, materials downloaded, videos watched, clicked links)

Number of patients deemed as having completed the intervention

Treatment as usual content in both arms from participants (number of sessions attended and content of sessions) and sites (initiatives implemented during the trial)

Safety (deaths, hospitalisations related to the intervention, RUSAEs)

Acceptability of the ACTION intervention to participants (acceptability and B\&N questionnaires)

Number, proportion and timing of therapist withdrawals and reasons for withdrawal

Qualitative study

Competency and fidelity of the delivery of the ACT intervention by NHS therapists (ACTKQ, ACT-FM, Procedural fidelity checklist)

"Proof-of-principle" exploration in outcome and process variables

Estimation of the Intracluster Correlation Coefficient

\section{Trial data collection}

We will collect data related to trial screening, eligibility, consent, contact details, and randomisation, questionnaire completion, withdrawal, and intervention adherence. 


\section{Participant Measures}

\section{Participant characteristics}

We will collect data on NHS number, date of birth, marital status, employment status, education, menopausal status, number of children, year of diagnosis, stage of cancer at diagnosis, breast cancer type (primary, secondary), breast cancer treatment received, co-morbidities, hormone therapy regimen, supportive therapies used, and previous exposure to psychotherapies.

Adherence Starts with Knowledge (ASK)-12 (45)

A 12 item, patient-report measure of barriers to medication adherence and adherence-related behavior.

McGill Quality of Life - Revised (46)

A 14-item, patient-report tool designed to measure physical well-being, physical symptoms, psychological symptoms, existential well-being and support, as well as overall quality of life of people with life-threatening illness.

Work and Social Adjustment Scale (47)

A 5-item self-report measure looking at how a specific illness/disorder impacts patient ability to function day to day with depression and/or anxiety as well as phobic disorders.

Hot Flash Related Daily Interference Scale (HFRDIS) (48)

A 10-item self-report questionnaire used to assess the impact of hot flashes on daily life and overall quality of life following breast cancer.

Multidimensional Assessment of Fatigue (MAF) (49)

A 16-item self-report scale that measures fatigue according to 4 dimensions; degree and severity, distress that it causes, timing of fatigue, and its impact on various activities of daily life.

PROMIS Pain Interference (50)

A 6-item self-report questionnaire measuring the consequences of pain over the last 7 days on relevant aspects of a person's life, including engagement with social, cognitive, emotional, physical and recreational activities.

Day-to-Day Impact of Vaginal Aging Questionnaire: DIVA (part C) (51)

Part C (scale focusing on sexual functioning) of the DIVA questionnaire contains 8 self-report items relating to the impact of vaginal symptoms on functioning and well-being.

Generalised Anxiety Disorder questionnaire (GAD-7) (52)

The GAD-7 is a 7 item self-report anxiety questionnaire designed to assess the patients' health status during the previous 2 weeks.

Patient Health Questionnaire- 9 (PHQ-9) (53)

The PHQ-9 is a 9 item self-reported scale providing a valid and reliable measure of depression severity.

Functional Assessment of Cancer Therapy - Endocrine Symptoms (54)

The FACT-ES is a self-report quality of life questionnaire for people with cancer, consisting of 4 sub scales which examine physical well-being with 7 items, social/family wellbeing with 7 items, emotional wellbeing with 6 items and functional wellbeing with 7 items as well as a series of items relating to additional concerns.

Valuing Questionnaire (VQ) (55)

A 10 item self-report questionnaire to measure aspects of psychological flexibility - mainly how consistently an individual has been living commensurate with their self-determined values.

\section{Acceptability}

Those participants recruited 3 months prior to the end of the recruitment phase and randomised to the ACT-based intervention will also complete items related to the acceptability of the intervention at 6 months post randomisation only. Items ask about the acceptability of the different components of ACTION and whether participants found it useful.

Borkovec and Nau Acceptability Questionnaire (B\&N) (56) 
Three items adapted from the B\&N will be collected, based on the wording used in the OBI study (57). This is an additional measure of acceptability and will be used within the progression criteria.

UK Cancer Costs Questionnaire (58)

We adapted the UK Cancer Costs Questionnaire to assess services accessed by the participants at baseline and follow up time points.

\section{Therapist measures}

ACT Knowledge Questionnaire (ACTKQ) (43)

The ACTKQ is an assessment of therapist knowledge of the principles of ACT. It has 16 items.

\section{Procedural fidelity checklist}

The procedural fidelity checklist was designed for the ACTION trial for therapists to self-rate whether or not they undertook crucial intervention procedures that were previously outlined in training and in the manual. It has 26 items, and is rated at the end of each of the four sessions.

ACT Fidelity Measure (ACT-FM) (44)

The ACT-FM is a measure of therapist fidelity to ACT principles when delivering treatment. There are 25 items capturing pre- and pro-scribed therapist behaviours when delivering ACT. The ACT-FM is scored when reviewing the therapy tapes of a clinician delivering ACT.

\section{Site measures}

\section{Intervention adherence}

Intervention adherence data will be collected from the delivering site, and will include the number of sessions attended and the number of participants in the group sessions.

\section{Treatment as usual monitoring}

Treatment as usual will be monitored at all recruitment sites. It will include data at the service level, including services offered, to allow for an assessment of treatment as usual. This data will be collected at the end of recruitment with sites asked to report treatment as usual throughout the recruitment phase.

\section{Sample size}

As the study is randomised and we wish to obtain a preliminary estimate of effectiveness in relation to demonstrating how the intervention affects medication adherence, we have used methods developed for phase II screening trials in oncology (59) in which preliminary and non-definitive randomised treatment comparisons are made, carefully adjusting the false-positive (a) and false-negative (ß) error rates so the target treatment effect is appropriate while the sample size remains restricted. Allowing for $25 \%$ loss to follow-up and using a 1 -sided t-test with a significance level of $20 \%$ will allow us to detect an effect size of 0.432 with $65 \%$ power using a sample of 80 patients. The sample size must be sufficient to establish consent and dropout rates (Objective i) and test trial protocols and acceptability (Objectives ii-iii). A sample size of $\mathrm{N}=40$ per arm will be sufficient to meet these aims (60-62).

\section{Nested qualitative study}

Following completion of the study, all participants assigned to the intervention group will be invited to participate in telephone interviews with a researcher. Interviews will focus on the acceptability of the intervention, trial procedures and outcome measures. We will seek to identify components of the intervention that were perceived as particularly helpful or unhelpful to participants. If aspects of the trial are considered unacceptable or unhelpful, questions will be asked to resolve some of these issues in preparation for a phase III RCT.

The therapists delivering the intervention will also be invited to participate in semi-structured telephone interviews. These will inquire about therapist's experiences of delivering the intervention, the practicability of the intervention (within NHS contexts), training, and ways to improve the programme.

\section{Analysis}

A detailed statistical analysis plan will be written before any analysis is undertaken. The analysis will focus on descriptive statistics and confidence interval estimation rather than formal hypothesis testing and no formal evaluation of safety or efficacy of the study interventions will be conducted. All analyses will be undertaken on the intention-to-treat population, with all participants included in the analysis according to their randomised allocation, and regardless of non-adherence to the intervention or withdrawal from the study. Final analysis will be conducted once all available outcome data is received.

Descriptive summaries will be presented overall, by arm and by site (where relevant) using appropriate frequencies and summary statistics. Outcome measures will be scored according to relevant scoring manuals. To generate evidence of proof of principle, the mean change from baseline in the three and six month ASK-12 scores in both arms will be reported, together with a range of confidence intervals around the main estimate to inform us as to the likelihood of where the 'true' estimate may lie. Analysis will adjust for the minimisation factors.

The trial is not powered to provide a precise estimate of the level of clustering relating to therapist effects, but we may be able to investigate this effect. If a sufficient number of therapists are involved in delivering the intervention, we will estimate the Intraclass Correlation Coefficient and produce a range of 
confidence intervals (e.g. $95 \%, 67 \%$ and $51 \%$ ) around this to inform the sample size of the definitive trial.

The semi-structured interviews will be analysed using thematic analysis (63).

\section{Progression Criteria}

Progression criteria will be used to judge whether it is feasible to progress to a larger study (Table 6). Analysis of progression criteria will take place during final analysis of the trial.

Table 6

Progression criteria for progression to a phase III RCT

\begin{tabular}{|c|c|c|c|}
\hline & Green & Amber & Red \\
\hline Meaning & $\begin{array}{l}\text { RCT is feasible No changes } \\
\text { needed }\end{array}$ & $\begin{array}{l}\text { RCT is feasible } \\
\text { following minor } \\
\text { changes }\end{array}$ & $\begin{array}{l}\text { RCT is not feasible } \\
\text { without major } \\
\text { changes }\end{array}$ \\
\hline \multicolumn{4}{|l|}{ Objective 1: Recruitment and follow-up } \\
\hline Eligible patients consent rate & $\geq 30 \%$ & $>10 \%$ & $<10 \%$ \\
\hline $\begin{array}{l}\text { Randomised patients adherence to intervention (\% attending the } \\
\text { individual session and at least one of the three group sessions) }\end{array}$ & $\geq 75 \%$ & $>40 \%$ & $<40 \%$ \\
\hline Loss to follow-up & $\leq 25 \%$ & $>25 \%$ & $>35 \%$ \\
\hline \multicolumn{4}{|l|}{ Objective 2: Acceptability } \\
\hline Average score across the three items on the $B \& N$ questionnaire & $\geq 6$ & $<6$ & $<4$ \\
\hline \multicolumn{4}{|l|}{ Objective 3: Competence and fidelity } \\
\hline Therapists achieving $\geq 50 \%$ on the ACTKQ & $100 \%$ & $\geq 50 \%,<100 \%$ & $<50 \%$ \\
\hline $\begin{array}{l}\text { Number of sessions scoring } \geq 80 \% \text { on procedural fidelity checklist } \\
\text { (Procedural fidelity) }\end{array}$ & $\geq 75 \%$ & $\geq 50 \%,<75 \%$ & $<50 \%$ \\
\hline Number of sessions scoring $\geq 39 \%$ on ACT-FM (ACT fidelity) & $\geq 75 \%$ & $\geq 50 \%,<75 \%$ & $<50 \%$ \\
\hline \multicolumn{4}{|l|}{ Objective 4: Proof of Principle } \\
\hline Change in adherence measured using ASK-12 & $\begin{array}{l}\text { Trend towards } \\
\text { improvement in the } \\
\text { intervention group }\end{array}$ & No obvious trend & $\begin{array}{l}\text { Worse scores in } \\
\text { intervention group }\end{array}$ \\
\hline
\end{tabular}

\section{Data management}

Data collection forms transferred to or from the CTRU will be coded with a study number (made up of the recruitment site code and the participant's unique sequential trial number), the participant's initials, and date of birth. Study data will be held securely on paper and electronically at the University of Leeds' CTRU, and appropriate processes put in place for the transfer, storage, restricted access, and disposal of personal information. Relevant Standard Operating Procedures, Guidelines and Work Instructions in relation to data management, processing, and analysis of data will be followed.

\section{Access to data}

Data will only be shared for participants who have given consent to use of their data for secondary research, and will only be made available in such a way that recipients cannot identify individuals by any reasonable likely means, and we will only share data for projects that are clearly in the public interest and compatible with the original purpose of the data processing. Requests to access trial data should be made to CTRU-DataAccess@leeds.ac.uk in the first instance.

\section{Independent Monitoring}

The Trial Steering Committee has responsibility for the oversight of the trial, including trial progress, adherence to protocol, participant safety and consideration of new information. The trial steering committee will be provided with reports prepared by the CTRU. It has been formed and consists of an independent chair (statistician), a clinical psychologist, a behavioural scientist, and two patient representatives. The Committee will meet annually as a minimum. As this is a pilot study and the level of risk involved is low with regard to patient safety there will be no data monitoring committee convened. The Trial Steering Group will adopt a safety function where it becomes necessary.

\section{Safety}

In this population, we expect episodes of acute illness, infection, new medical problems and deterioration of existing medical problems to occur. These could result in prolonged hospitalisation, hospital re-admission, significant or permanent disability or incapacity, or death. In recognition of this, events fulfilling the definition of a serious adverse event will not be reported in this study unless the event resulted from administration of any research procedure and fulfils definition of a related and unexpected serious adverse event (RUSAE). Deaths will be recorded, but not subjected to expedited reporting. Hospitalisations will be recorded by contacting sites at the end of the study. Pregnancies will also be reported to the CTRU. 
All RUSAEs occurring from the date of consent until the end of researcher contact will be reviewed by the Chief Investigator and be subject to expedited reporting to the Sponsor and the main research ethics committee by the CTRU on behalf of the Cl within 15 days.

Concerns over patient safety will be monitored by reviewing responses to pre agreed 'red flags' assigned to the PHQ-9 questionnaire (if question 9 is answered 1-3 at any time point). If this occurs in the baseline questionnaire, the Chief Investigator (or delegate) will be notified within 2 working days of receipt at the CTRU and will contact the patient to follow up and repeat the screening questionnaire with them to make sure they are a) not a danger to themselves or others, and b) still eligible. If this response is given during the follow up questionnaires the Chief Investigator (or delegate) will be notified within 2 working days of receipt at the CTRU and will report this to the PI at the recruiting site. If the patient is still receiving trial treatment the psychology team at the central delivery site will be informed and will contact the patient and repeat the screening questionnaire as above.

\section{Discussion}

Non-adherence to AET is a major problem among women with breast cancer (4), and is linked with an increased risk of cancer-specific and all-cause mortality (5-12). Women who are unable to adhere to their adjuvant treatment are likely to need additional support to address the challenges they experience with this behaviour, and with their broader quality of life. A number of barriers to medication use have been reported in this setting, including medication side-effects, psychological distress and a lack of social support $(4,15)$. The ACTION intervention aims to address these barriers through a codeveloped remotely delivered group-based psychotherapy programme, informed by ACT.

Prior to testing the ACTION intervention in a phase III RCT, additional information is needed across a range of areas. This includes exploring the acceptability of the intervention to participants and NHS staff, examining the extent to which NHS therapists can deliver the intervention with fidelity, and demonstrating the feasibility of recruiting and retaining patients in this setting. This exploratory multi-centre pilot trial with a nested qualitative study aims to provide information to inform our future decision as to the feasibility of a definitive trial. While we are not attempting to evaluate the efficacy of the intervention, it will be our first opportunity to examine signals of efficacy of the intervention on our planned outcome measures.

The ACTION intervention advances our knowledge of ACT as it is among the first programmes to be tested within an RCT to focus on medication decisionmaking and quality of life. Although ACT has been proposed as a means to enhance adherence to treatments, and case series have emerged in recent years, there have been few comprehensive assessments of the approach $(29,37,38)$. Also novel within the methodology of ACTION is the comprehensive focus on therapist competency and fidelity to ACT principles and ACTION procedures. For psychological interventions that require skill in implementation, this pilot offers an important opportunity to understand the competency and fidelity of delivering the intervention. This information will be used to improve training, supervision and the suitability of the intervention for clinical practice.

In response to the COVID-19 pandemic, our intervention will involve remotely delivered therapy, using video conferencing software. This alteration to original plans brings new opportunities with regard to widespread implementation and uptake. Participants do not need to travel to attend and capacity is not limited by the availability of rooms within hospitals. We anticipate that it may also increase access to specific groups who may be unable to access inperson support as readily, including rural populations, those lacking transport links, and younger populations who have returned to work following treatment. A remotely delivered approach may also have limitations, some of which are beginning to emerge following evaluations of clinical care during the COVID-19 pandemic (64). Potential problems include difficulties building therapeutic relationships as easily, technical challenges in using unfamiliar software and hardware, and the possibility of disenfranchising women who cannot access the internet or a private space to participate fully within groups. These issues will be carefully considered using the data provided within the pilot trial, and may be most apparent within the nested qualitative study.

In conclusion, this multi-centre randomised controlled external pilot trial will examine the feasibility of delivering a co-developed psychotherapy intervention to support medication adherence and quality of life in women with breast cancer. If the pilot data indicate it is feasible, ACTION can be evaluated in a larger phase III RCT.

\section{Study status}

Enrolling. Recruitment began 04/2021. Approximate date recruitment will be completed: 09/2022. Latest protocol version approved 5.0_030221 on 05/02/2021 by Yorkshire \& The Humber - South Yorkshire Research Ethics Committee (20/YH/0104).

\section{Abbreviations}

Acceptance and Commitment Therapy (ACT)

Adjuvant Endocrine therapy (AET)

ACT Fidelity Measure (ACT-FM)

ACT Knowledge Questionnaire (ACTKQ)

Acceptance and Commitment Therapy Intervention (ACTION)

Adherence Starts with Knowledge (ASK)

Aromatase Inhibitors (Als) 
Borkovec and Nau (B\&N)

Clinical Trials Research Unit (CTRU)

Coronavirus Disease-19 (COVID-19)

Day-to-Day Impact of Vaginal Aging Questionnaire (DIVA)

Functional Assessment of Cancer Therapy - Endocrine Symptoms (FACT-ES)

Generalised Anxiety Disorder questionnaire (GAD-7)

Hot Flash Related Daily Interference Scale (HFRDIS)

Health and Care Professional Council (HCPC)

Human Immunodeficiency Virus (HIV)

Multidimensional Assessment of Fatigue (MAF)

National Health Service (NHS)

National Research Ethics Service (NRES)

Oestrogen receptor positive (ER+)

Patient Health Questionnaire- 9 (PHQ-9)

Randomised Controlled Trial (RCT)

Related and unexpected serious adverse event (RUSAE)

Selective Oestrogen Receptor Modulators (SERMs)

Valuing Questionnaire (VQ)

Work and Social Adjustment Scale (WSAS)

\section{Declarations}

\section{Study amendments}

Not applicable.

\section{Ethics approval and consent to participate}

This study was approved by Yorkshire \& The Humber - South Yorkshire Research Ethics Committee on 05/02/2021 (20/YH/0104).

\section{Consent for publication}

Not applicable.

\section{Availability of data and materials}

Not applicable.

\section{Competing interests}

The authors declare that they have no competing interests

\section{Funding}

The study was funded by Yorkshire Cancer Research (funder ref: L417) (PI: SS and CG). SS is also funded by a Yorkshire Cancer Research Fellowship. The funder had no role in the design of the study, data collection, analysis, interpretation of data, and in the writing of this manuscript.

\section{Authors' contributions}

SS, RE, LH, JC, SH, EM, JM, CD, VN, RF, RN, GV, AM, MC, CG designed the study

RE, JC, SH, EM, CH, SueH, JM, VN, JN, SB, AP, MC managed and collected the data 
SS, RE, LH, JC, SH, EM, JN, SB, AP, RF, RN, GV, AF, MC, CG analysed and interpreted the data

SS, RE, LH, SH, EM, JM, CO, AF, MC, CG contributed to writing the first draft of the manuscript

SS, RE, LH, JC, SH, EM, JM, CD, VN, CH, SueH, JN, SB, AP, RF, RN, GV, AF, MC, CG reviewed the manuscript draft and approved the final manuscript.

\section{Acknowledgements}

We acknowledge the support of Cheryl Malpass, Julie Saritag, Rachel Brown, Gita Robson, lan Lorentz, Lucy Holmes and Nicky Bould, Anita Wraith, Adrienne Vince, Jessica Lane, Robbie Foy, Duncan Petty and Andrew Proctor.

\section{References}

1. Forouzanfar MH, Foreman KJ, Delossantos AM, Lozano R, Lopez AD, Murray CJL, et al. Breast and cervical cancer in 187 countries between 1980 and 2010: a systematic analysis. The Lancet. 2011;378(9801):1461-84.

2. Smittenaar CR, Petersen KA, Stewart K, Moitt N. Cancer incidence and mortality projections in the UK until 2035. Br J Cancer. 2016;115(9):114755 .

3. Early and locally advanced breast cancer: diagnosis and management. NICE. Available from: https://www.nice.org.uk/guidance/ng101

4. Murphy CC, Bartholomew LK, Carpentier MY, Bluethmann SM, Vernon SW. Adherence to adjuvant hormonal therapy among breast cancer survivors in clinical practice: a systematic review. Breast Cancer Res Treat. 2012;134(2):459-78.

5. Makubate B, Donnan PT, Dewar JA, Thompson AM, McCowan C. Cohort study of adherence to adjuvant endocrine therapy, breast cancer recurrence and mortality. Br J Cancer. 2013;108(7):1515-24.

6. McCowan C, Wang S, Thompson AM, Makubate B, Petrie DJ. The value of high adherence to tamoxifen in women with breast cancer: a community-based cohort study. Br J Cancer. 2013;109(5):1172-80.

7. Dezentjé VO, van Blijderveen NJC, Gelderblom H, Putter H, van Herk-Sukel MPP, Casparie MK, et al. Effect of concomitant CYP2D6 inhibitor use and tamoxifen adherence on breast cancer recurrence in early-stage breast cancer. J Clin Oncol. 2010;28(14):2423-9.

8. Ma AMT, Barone J, Wallis AE, Wu NJ, Garcia LB, Estabrook A, et al. Noncompliance with adjuvant radiation, chemotherapy, or hormonal therapy in breast cancer patients. Am J Surg. 2008;196(4):500-4.

9. Ulcickas Yood M, Owusu C, Buist DSM, Geiger AM, Field TS, Thwin SS, et al. Mortality Impact of Less-than-Standard Therapy in Older Breast Cancer Patients. J Am Coll Surg. 2008;206(1):66-75.

10. Winn AN, Dusetzina SB. The association between trajectories of endocrine therapy adherence and mortality among women with breast cancer. Pharmacoepidemiol Drug Saf. 2016;25(8):953-9.

11. Chirgwin JH, Giobbie-Hurder A, Coates AS, Price KN, Ejlertsen B, Debled M, et al. Treatment Adherence and Its Impact on Disease-Free Survival in the Breast International Group 1-98 Trial of Tamoxifen and Letrozole, Alone and in Sequence. J Clin Oncol. 2016;34(21):2452-9.

12. McCowan C, Shearer J, Donnan PT, Dewar JA, Crilly M, Thompson AM, et al. Cohort study examining tamoxifen adherence and its relationship to mortality in women with breast cancer. Br J Cancer. 2008;99(11):1763-8.

13. Harrison A, Graham C, McCraken L. Treatment predictors in chronic pain: predictors, models and a role of psychological flexibility. In: Cheatle M, Fine PG, editors. Facilitating treatment adherence in pain medicine. Oxford University Press; 2017, p8-30

14. Kardas P, Lewek P, Matyjaszczuk M. Determinants of patient adherence: a review of systematic reviews. Front Pharmacol. $2013 ; 4: 91$.

15. Cahir C, Guinan E, Dombrowski SU, Sharp L, Bennett K. Identifying the determinants of adjuvant hormonal therapy medication taking behaviour in women with stages I-III breast cancer: A systematic review and meta-analysis. Patient Educ Couns. 2015;98(12):1524-39.

16. Hunter MS, Grunfeld EA, Mittal S, Sikka P, Ramirez A-J, Fentiman I, et al. Menopausal symptoms in women with breast cancer: prevalence and treatment preferences. Psychooncology. 2004;13(11):769-78.

17. Cella D, Fallowfield LJ. Recognition and management of treatment-related side effects for breast cancer patients receiving adjuvant endocrine therapy. Breast Cancer Res Treat. 2008;107(2):167-80.

18. Atkins L, Fallowfield L. Intentional and non-intentional non-adherence to medication amongst breast cancer patients. Eur J Cancer. 2006;42(14):2271-6. 
19. Nestoriuc Y, Blanckenburg P von, Schuricht F, Barsky AJ, Hadji P, Albert U-S, et al. Is it best to expect the worst? Influence of patients' side-effect expectations on endocrine treatment outcome in a 2-year prospective clinical cohort study. Ann Oncol; 2016; 27(10): 1909-15.

20. Smith SG, Sestak I, Howell A, Forbes J, Cuzick J. Participant-Reported Symptoms and Their Effect on Long-Term Adherence in the International Breast Cancer Intervention Study I (IBIS I). J Clin Oncol. 2017;35(23): 2666-2673.

21. Sestak I, Smith SG, Howell A, Forbes J, Cuzick J. Early participant-reported symptoms as predictors of adherence to anastrozole in the International Breast Cancer Intervention Studies II. Ann Oncol. 2018; 29(2): 504-509.

22. Castel LD, Hartmann KE, Mayer IA, Saville BR, Alvarez J, Boomershine CS, et al. Time course of arthralgia among women initiating aromatase inhibitor therapy and a postmenopausal comparison group in a prospective cohort. Cancer. 2013;119(13):2375-82.

23. Hurtado-de-Mendoza A, Cabling ML, Lobo T, Dash C, Sheppard VB. Behavioral Interventions to Enhance Adherence to Hormone Therapy in Breast Cancer Survivors: A Systematic Literature Review. Clin Breast Cancer. 2016;16(4):247-255.e3.

24. Finitsis DJ, Vose BA, Mahalak JG, Salner AL. Interventions to promote adherence to Endocrine Therapy among breast cancer survivors: A metaanalysis. Psychooncology. 2019; 28(2): 255-263.

25. Heiney SP, Parker PD, Felder TM, Adams SA, Omofuma 00, Hulett JM. A systematic review of interventions to improve adherence to endocrine therapy. Breast Cancer Res Treat. 2019; 173(3): 499-510.

26. McCracken LM, Morley S. The psychological flexibility model: a basis for integration and progress in psychological approaches to chronic pain management. J Pain. 2014;15(3):221-34.

27. Harrison A, Scott W, Timmins L, Graham CD, Harrison AM. Investigating the potentially important role of psychological flexibility in adherence to antiretroviral therapy in people living with HIV. AIDS Care. 2021;33(3):337-46.

28. Graham CD, Gouick J, Krahé C, Gillanders D. A systematic review of the use of Acceptance and Commitment Therapy (ACT) in chronic disease and long-term conditions. Clin Psychol Rev. 2016;46:46-58.

29. Hadlandsmyth K. Proposing an Acceptance and Commitment Therapy intervention to promote improved diabetes management in adolescents: A treatment conceptualization. Int J Behav Consult Ther. 20130610;7(4):12.

30. Fernandes-James C, Graham CD, Batterham AM, Harrison SL. Association of psychological flexibility with engagement in pulmonary rehabilitation following an acute exacerbation of chronic obstructive pulmonary disease. Chron Respir Dis. 2019. 16:1479973119880893.

31. Hulbert-Williams NJ, Storey L. Psychological flexibility correlates with patient-reported outcomes independent of clinical or sociodemographic characteristics. Support Care Cancer. 2016;24(6):2513-21.

32. Hann KEJ, McCracken LM. A systematic review of randomized controlled trials of Acceptance and Commitment Therapy for adults with chronic pain: Outcome domains, design quality, and efficacy. J Context Behav Sci. 2014;3(4):217-27.

33. Feros DL, Lane L, Ciarrochi J, Blackledge JT. Acceptance and Commitment Therapy (ACT) for improving the lives of cancer patients: a preliminary study. Psychooncology. 2013;22(2):459-64.

34. Rost AD, Wilson K, Buchanan E, Hildebrandt MJ, Mutch D. Improving Psychological Adjustment Among Late-Stage Ovarian Cancer Patients: Examining the Role of Avoidance in Treatment. Cogn Behav Pract. 2012;19(4):508-17.

35. Johns SA, Stutz PV, Talib TL, Cohee AA, Beck-Coon KA, Brown LF, et al. Acceptance and commitment therapy for breast cancer survivors with fear of cancer recurrence: A 3-arm pilot randomized controlled trial. Cancer. 2020;126(1):211-8.

36. Zhao C, Lai L, Zhang L, Cai Z, Ren Z, Shi C, et al. The effects of acceptance and commitment therapy on the psychological and physical outcomes among cancer patients: A meta-analysis with trial sequential analysis. J Psychosom Res. 2021;140:110304.

37. Moitra E, Herbert JD, Forman EM. Acceptance-based behavior therapy to promote HIV medication adherence. AIDS Care. 2011;23(12):1660-7.

38. Hoefnagels JW, Fischer K, Bos RAT, Driessens MHE, Meijer SLA, Schutgens REG, et al. A feasibility study on two tailored interventions to improve adherence in adults with haemophilia. Pilot Feasibility Stud. 2020;6(1):189.

39. Hall LH, Clark J, Smith S, Graham CD. Patient and healthcare professional co-development of an Acceptance and Commitment Therapy intervention to support Hormone Therapy decision-making and quality of life in women with breast cancer. submitted;

40. Chan A-W, Tetzlaff JM, Altman DG, Laupacis A, Gøtzsche PC, Krleža-Jerić K, et al. SPIRIT 2013 statement: defining standard protocol items for clinical trials. Ann Intern Med. 2013;158(3):200-7. 
41. Eldridge SM, Chan CL, Campbell MJ, Bond CM, Hopewell S, Thabane L, et al. CONSORT 2010 statement: extension to randomised pilot and feasibility trials. BMJ. 2016;355:i5239.

42. Hoffmann TC, Glasziou PP, Boutron I, Milne R, Perera R, Moher D, et al. Better reporting of interventions: template for intervention description and replication (TIDieR) checklist and guide. BMJ. 2014;348:g1687.

43. Richards R, Oliver JE, Morris E, Aherne K, lervolino AC, Wingrove J. Acceptance and Commitment Therapy training for clinicians: an evaluation. Cogn Behav Ther. 2011;4(3):114-21.

44. O'Neill L, Latchford G, McCracken L, Graham CD. The Development of the Acceptance and Commitment Therapy Fidelity Measure (ACT-FM): A Delphi Study and Field Test. J Context Behav Sci. 2019; 14, 111-118.

45. Matza LS, Park J, Coyne KS, Skinner EP, Malley KG, Wolever RQ. Derivation and validation of the ASK-12 adherence barrier survey. Ann Pharmacother. 2009;43(10):1621-30.

46. Cohen SR, Sawatzky R, Russell LB, Shahidi J, Heyland DK, Gadermann AM. Measuring the quality of life of people at the end of life: The McGill Quality of Life Questionnaire-Revised. Palliat Med. 2017;31(2):120-9.

47. Mundt JC, Marks IM, Shear MK, Greist JH. The Work and Social Adjustment Scale: a simple measure of impairment in functioning. Br J Psychiatry. 2002;180:461-4.

48. Carpenter JS. The Hot Flash Related Daily Interference Scale: a tool for assessing the impact of hot flashes on quality of life following breast cancer. J Pain Symptom Manage. 2001;22(6):979-89.

49. Belza BL, Henke CJ, Yelin EH, Epstein WV, Gilliss CL. Correlates of fatigue in older adults with rheumatoid arthritis. Nurs Res. 1993;42(2):93-9.

50. Amtmann D, Cook KF, Jensen MP, Chen W-H, Choi S, Revicki D, et al. Development of a PROMIS item bank to measure pain interference. PAIN. 2010;150(1):173-82.

51. Huang AJ, Gregorich SE, Kuppermann M, Nakagawa S, Van Den Eeden SK, Brown JS, et al. The Day-to-Day Impact of Vaginal Aging Questionnaire: A Multidimensional Measure of the Impact of Vaginal Symptoms on Functioning and Well-being in Postmenopausal Women. Menopause. 2015;22(2):144-54.

52. Spitzer RL, Kroenke K, Williams JBW, Löwe B. A Brief Measure for Assessing Generalized Anxiety Disorder: The GAD-7. Arch Intern Med. 2006;166(10):1092-7.

53. Spitzer RL, Kroenke K, Williams JB. Validation and utility of a self-report version of PRIME-MD: the PHQ primary care study. Primary Care Evaluation of Mental Disorders. Patient Health Questionnaire. JAMA. 1999;282(18):1737-44.

54. Fallowfield LJ, Leaity SK, Howell A, Benson S, Cella D. Assessment of quality of life in women undergoing hormonal therapy for breast cancer: Validation of an endocrine symptom subscale for the FACT-B. Breast Cancer Res Treat. 1999;55(2):187-197.

55. Smout M, Davies M, Burns N, Christie A. Development of the Valuing Questionnaire (VQ). J Context Behav Sci. 2014;3(3):164-72.

56. Borkovec TD, Nau SD. Credibility of analogue therapy rationales. J Behav Ther Exp Psychiatry. 1972;3(4):257-60.

57. Pincus T, Anwar S, McCracken LM, McGregor A, Graham L, Collinson M, et al. Delivering an Optimised Behavioural Intervention (OBI) to people with low back pain with high psychological risk; results and lessons learnt from a feasibility randomised controlled trial of Contextual Cognitive Behavioural Therapy (CCBT) vs. Physiotherapy. BMC Musculoskelet Disord. 2015;16:147.

58. UK Cancer Costs Questionnaire. Available from: https://blogs.ed.ac.uk/ukcc/

59. Rubinstein LV, Korn EL, Freidlin B, Hunsberger S, Ivy SP, Smith MA. Design issues of randomized phase II trials and a proposal for phase II screening trials. J Clin Oncol. 2005;23(28):7199-206.

60. Lancaster GA, Dodd S, Williamson PR. Design and analysis of pilot studies: recommendations for good practice. J Eval Clin Pract. 2004;10(2):307-12.

61. Billingham SAM, Whitehead AL, Julious SA. An audit of sample sizes for pilot and feasibility trials being undertaken in the United Kingdom registered in the United Kingdom Clinical Research Network database. BMC Med Res Methodol. 2013;13:104.

62. Whitehead AL, Julious SA, Cooper CL, Campbell MJ. Estimating the sample size for a pilot randomised trial to minimise the overall trial sample size for the external pilot and main trial for a continuous outcome variable. Stat Methods Med Res. 2016;25(3):1057-73.

63. Braun V, Clarke V. Using thematic analysis in psychology. Qual Res Psychol. 2006;3:77-101. 
64. Liberati E, Richards N, Parker J, Willars J, Scott D, Boydell N, et al. Remote care for mental health: qualitative study with service users, carers and staff during the COVID-19 pandemic. BMJ Open. 2021;11(4):e049210.

\section{Figures}

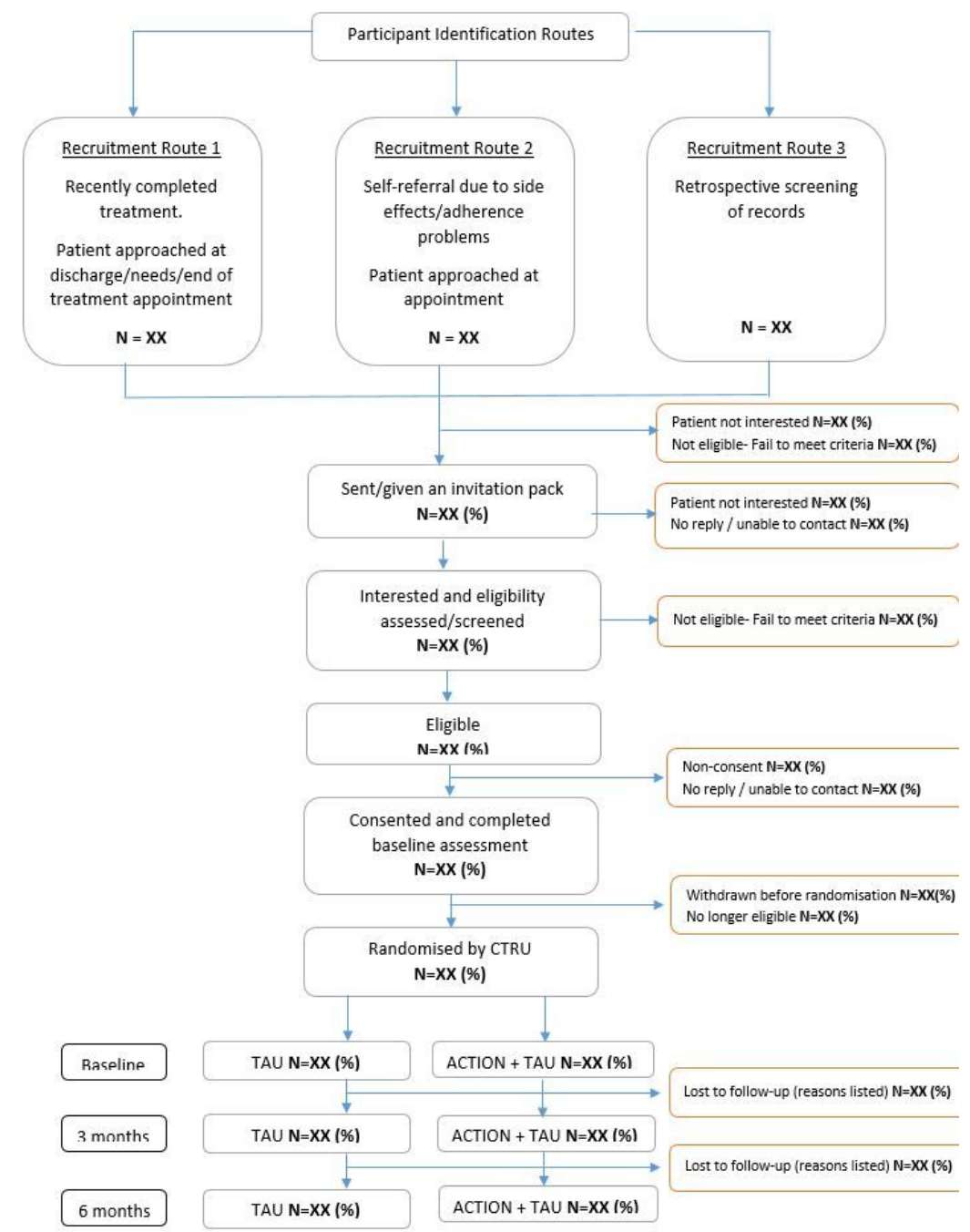

Figure 1

Participant Identification Routes 